HIV healthcare professional (HCP) beliefs and practices around sharing diverse types of service user and clinic-generated data across multiple contexts.

Methods During February-October 2020, we conducted 14 semi-structured interviews with HCPs working in a large UK HIV outpatient service. Participants engaged in a card sorting task, sorting 33 data types routinely shared in HIV care into categories (comfortable/not comfortable/not sure) across three data-sharing contexts: (a) service users to HCPs in consultations; (b) HCPs to GPs; (c) HCPs to non-HIV HCPs. Data were analysed thematically.

Results Over half (57\%) of participants were female; 57\% were doctors. Participants had worked in HIV for 12 years on average.

HCPs were comfortable with a wide range of data being shared with them by service users. Across all sharing contexts, HCPs were uncomfortable with sharing of service user photographs, perceiving them as not routinely shared, unnecessary, and potentially risking inadvertent sharing of inappropriate content. HCPs were comfortable sharing data with GPs and other non-HIV HCPs in two broad categories: (a) demographic data (e.g. age) and (b) non-sensitive data related to general health (e.g. sleep). HCPs were less comfortable sharing sensitive information about HIV status, sexual health, behaviour and identity. Service user consent and relevance of data to sharing context were key determinants of data-sharing comfort.

Conclusion Understanding the context of data-sharing in HIV care is increasingly important given the shift to remote consultations and expectations for self-management. We demonstrate the complex interplay of data types, relationship dynamics, and contexts of care provision that shape the data-sharing ecosystem in HIV care. Developing guidance on the sharing of service user and clinic-generated data in HIV care must account for these complexities.

\section{P209 SWITCHING TO DORAVIRINE IN CART EXPERIENCED PATIENTS: EFFECTIVE, HIGHLY TOLERATED, AND COST SAVING. A RETROSPECTIVE COHORT STUDY}

${ }^{1} \mathrm{~V}$ Lanting*, ${ }^{1} \mathrm{P}$ Oosterhof, ${ }^{1} \mathrm{D}$ Ait Moha, ${ }^{1} \mathrm{G}$ Van den Berk, ${ }^{1,2} \mathrm{~K}$ Brinkman. ${ }^{1}$ OLVG hospital, Amsterdam, The Netherlands; ${ }^{2}$ University of Amsterdam, Amsterdam, The Netherlands

\subsection{6/sextrans-2021-sti.298}

Background Doravirine is a NNRTI with demonstrated effect as third agent in treatment naive and experienced HIV patients.

Aim To evaluate outcomes of cART experienced patients switching their therapy to DOR/TDF/3TC or doravirine based regimens, with focus on efficacy, tolerability and costs.

Methods Baseline characteristics like treatment history, and laboratory values were retrospectively collected with follow up data at 6 months. If patients stopped therapy, reasons were evaluated. Baseline and follow-up data were compared in patients that continued therapy using IBM SPSS. Potential cost savings compared to prior cART were estimated for the cohort that continued DOR/TDF/3TC for follow-up period and extrapolated to the average per year.

Results A total of 377 patients (91\% men) were included that completed outpatient clinic visit or/and laboratory data at 6 months: $97.1 \%$ switched to Doravirine/tenofovir/lamivudine (DOR/TDF/3TC) and $2.9 \%$ to other doravirine based regimens. Doravirine was prematurely stopped in 58/377 patients (15.4\%). Reason to stop was a patient-reported adverse event in $43 / 377$ patients $(11.4 \%)$; most frequently gastrointestinal (3.8\%) or insomnia/sleep disturbances $(3.4 \%)$. Other reasons were virologic failure $(0,8 \%)$ with only 1 case demonstrating resistance (V106VA, V108VI), decreased renal function $(0,8 \%)$, and ALAT grade $2(0,5 \%)$.

Within the group that continued doravirine grade 1 ALAT elevation was more frequently observed at 6 months compared to baseline $(14.2 \%$ vs. $7.9 \%(p=0.004), n=318)$; no grade $2 /$ $3 / 4$ increase was observed. A decrease in LDL-c was seen $(3.50( \pm 1.10) \quad \mathrm{mmol} / \mathrm{L}$ vs. $2.97( \pm 0.79) \quad \mathrm{mmol} / \mathrm{L} ; \mathrm{n}=42$, $\mathrm{p}<0.001)$. No change in creatinine levels was observed. The switch in 319 patients that continued therapy saved 33\% in costs with an average saving of $€ 3,130$ euros per patient/year $(€ 1$ million total).

Conclusion Doravirine is a suitable therapy for maintenance treatment and shows enormous savings. In addition, our data shows that it is also effective and well tolerated by $85 \%$ in our patient group.

\section{P211 CHANGES IN SEXUAL BEHAVIOR DURING COVID-19 AMONG MEN WHO HAVE SEX WITH MEN IN A SEXUAL NETWORK STUDY}

${ }^{1} \mathrm{M}$ Spahnie*, ${ }^{1} \mathrm{~A}$ Matthews, ${ }^{1} \mathrm{D}$ Kiss, ${ }^{1} \mathrm{~L}$ Jones, ${ }^{2} \mathrm{C}$ Copen, ${ }^{2} \mathrm{~J}$ Rushmore, ${ }^{2} \mathrm{~K}$ Bernstein, ${ }^{1} \mathrm{~J}$ Ricks, ${ }^{1} \mathrm{~W}$ Miller, ${ }^{3} \mathrm{~A}$ Norris Turner. ${ }^{1}$ College of Public Health, The Ohio State University, Columbus, USA; ${ }^{2}$ Division of STD Prevention, Centers for Disease Control and Prevention, Atlanta, USA; ${ }^{3}$ College of Medicine, The Ohio State University, Columbus, USA

\subsection{6/sextrans-2021-sti.299}

Background As part of an ongoing sexual network study, we assessed the impact of COVID-19 on sexual behavior of men who have sex with men (MSM).

Methods The Columbus, Ohio arm of the multi-site Network Epidemiology of Syphilis Transmission (NEST) study is following 241 MSM over two years. Participants attend quarterly visits with behavioral surveys and HIV/STI testing. In April 2020, we implemented an additional survey to measure the impact of COVID-19 on sexual behavior. Our analysis compared participants' reported sexual behavior during two periods: April-July 2020 (T1) and August-December 2020 (T2).

Results Of the 200 participants who completed at least one COVID-19 survey in either time period, 10 (5\%) self-reported a previous COVID-19 diagnosis. We compared behaviors reported by 174 respondents who submitted surveys in both time periods. During T1, about half $(46 \%)$ said they felt more anxious about sex since COVID-19; anxiety was similar during T2 (43\%). Many reported engaging in online sexual activities, though we saw no meaningful differences by time period for starting or increasing chatting on hookup apps ( $\mathrm{T} 1=30 \%, \mathrm{~T} 2=30 \%)$, sexting $(\mathrm{T} 1=30 \%, \mathrm{~T} 2=25 \%)$, or video chat sex $(\mathrm{T} 1=16 \%, \mathrm{~T} 2=14 \%)$. Watching pornography was reported more often in T1 than T2 (53\% vs. 42\%). Nearly a third of respondents $(30 \%)$ in T1 reported that they were not having sex; this decreased to $17 \%$ in $\mathrm{T} 2$. Compared to prepandemic behavior, in T1 a majority of participants (74\%) said they were less likely to have sex with a new partner, compared to $61 \%$ during T2.

Conclusion During COVID-19, nearly half of MSM reported feeling more anxious about sex, and many turned to online activities in lieu of in-person sex. However, when comparing 
the early months of the pandemic to later months of 2020, more participants are resuming in-person sex, and willingness to have sex with new partners also rose.

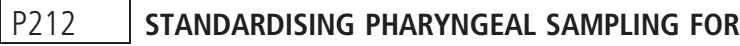 CLINICIAN-TAKEN GONORRHOEA CULTURE SPECIMENS}

${ }^{1} S$ Stuart* ${ }^{1,2} \mathrm{D}$ Richardson, ${ }^{1,2} \mathrm{C}$ Iwuji, ${ }^{1,2} \mathrm{~S}$ Soni. 'Brighton and Sussex Medical School, Brighton, UK; ${ }^{2}$ Brighton and Sussex University Hospitals NHS Trust, Brighton, UK

\subsection{6/sextrans-2021-sti.300}

Introduction The pharynx is an important site with respect to transmission and development of antimicrobial resistance (AMR) to gonorrhoea particularly in men who have sex with men. Maintaining culture-based sensitivity testing is important as part of wider efforts to prevent transmission of high level AMR strains of gonorrhoea. The sensitivity of gonorrhoea culture at the pharynx is low and is dependent on several factors including sampling technique by clinicians. There is no consensus on the length of time taken to sample the pharynx for culture. We aimed to explore knowledge of pharyngeal sampling among sexual health clinicians to gain a consensus on optimal time for pharyngeal sampling for gonorrhoea culture specimens.

Method An online anonymous survey was sent to clinicians in our local sexual health network. We included pictorial diagrams of the pharynx and asked clinicians which sites in the pharynx they sample and to estimate how long for when taking gonorrhoea culture specimens.

Result 110 clinicians responded to the survey of whom 98 regularly take pharyngeal gonorrhoea cultures specimens: 54 $(55 \%)$ had more than 10-years of experience in sexual health. $82(84 \%)$ sample the tonsils, $75(77 \%)$ sample the posterior pharyngeal wall and 94(96\%) sample either the tonsils or the posterior pharyngeal wall. $30(31 \%)$ always attempt to elicit the gag reflex and $52(53 \%)$ do not. The average time taken sampling the pharynx was $4.63(\mathrm{SD} \pm 2.04)$ seconds. There was no significant difference in time taken to sample the pharynx according to years of experience $(4.7(\mathrm{SD} \pm 2.02) \mathrm{v} 4.6(\mathrm{SD} \pm 2.3)$, $\mathrm{p}=0.45)$.

Conclusion Experienced sexual health clinicians are mostly appropriately sampling the tonsils and posterior pharyngeal wall for gonorrhoea culture specimens, and the median time taken to sample the pharynx was 4.63 seconds. Standardisation of pharyngeal sampling for the pharynx for gonorrhoea culture specimens could improve the sensitivity of culture and educational tools for clinic staff to support this are needed.

\section{P213 IDENTIFICATION OF TRICHOMONAS VAGINALIS 5- NITROIMIDAZOLE RESISTANCE TARGETS TO INFORM FUTURE DRUG DEVELOPMENT}

${ }^{1} \mathrm{~K}$ Graves* ${ }^{*},{ }^{1} \mathrm{~J}$ Sharma, ${ }^{1,2} \mathrm{C}$ Reily, ${ }^{3} \mathrm{~W}$ Secor, ${ }^{2} \mathrm{~J}$ Novak, ${ }^{1} \mathrm{C}$ Muzny. ${ }^{1}$ Department of Medicine, University of Alabama at Birmingham, Birmingham, USA; ${ }^{2}$ Department of Microbiology, University of Alabama at Birmingham, Birmingham, USA; ${ }^{3}$ Division of Parasitic Diseases and Malaria, Center for Global Health, Centers for Disease Control and Prevention, Atlanta, USA

10.1136/sextrans-2021-sti.301

Background Trichomonas vaginalis is the most common nonviral sexually transmitted infection. 5-nitroimidazoles (i.e., metronidazole [MTZ] and tinidazole) are the only FDAapproved medications. Resistance to 5- nitroimidazoles has been observed in $5-10 \%$ of cases, but may be rising. MTZ was first introduced in the early 1960 s to treat $T$. vaginalis infections, and resistance to MTZ developed rapidly within a couple of years. This observation suggests that the potential for MTZ resistance is likely encoded in the genome of T. vaginalis. We aimed to analyze gene-expression patterns of MTZresistant and MTZ-sensitive T. vaginalis isolates to gain insight into resistance mechanisms.

Methods T. vaginalis isolates (4 MTZ-resistant [minimal lethal concentration; MLC $>50 \mu \mathrm{g} / \mathrm{ml}$ ] and 4 MTZ-sensitive [MLC $<25 \mu \mathrm{g} / \mathrm{ml}]$ ) were grown in triplicate in Diamond's TYM medium. MTZ susceptibility testing confirmed MTZ MLCs of T. vaginalis isolates. RNA sequencing (RNAseq) and bioinformatics analyses were used to identify differentially expressed genes (DEGs) in MTZ-resistant vs. sensitive isolates. Subsequent qPCR was used to confirm and extend RNAseq data and gene targets related to 5-nitroimidazole resistance.

Results RNAseq identified DEGs in MTZ-resistant vs. sensitive isolates. DEGs from MTZ-resistant isolates included those involved in metabolic pathways relevant to 5-nitroimidazole resistance such as energy production, drug activation, and oxygen-scavenging. Other DEGs included those encoding transcription factors, ribosomal proteins, and other metabolic enzymes. RT-qPCR experiments confirmed reduced expression of genes encoding ferredoxin $(\mathrm{Fdx})$ and flavin reductase 1 (FR1) in MTZ-resistant T. vaginalis isolates as compared to MTZ-sensitive isolates.

Conclusion In this study, we identified several DEGs in T. vaginalis isolates resistant to MTZ. Further studies with large number of isolates representing a broad range of MTZ-susceptibility patterns are needed to identify genes that may represent new targets for future drug development.

\section{P215 EFFECTIVENESS OF COMMUNITY-BASED HEALTH PROMOTION CAMPAIGN METHODS ON CHLAMYDIA SCREENING UPTAKE IN YOUNG PEOPLE: A MIXED- METHODS SYSTEMATIC REVIEW}

${ }^{1} \mathrm{E}$ Pearce*, ${ }^{1} \mathrm{~K}$ Jolly, ${ }^{1} \mathrm{D}$ Moore, ${ }^{1} \mathrm{~B}$ Harris, ${ }^{1} \mathrm{~A}$ Adriano, ${ }^{2} \mathrm{~J}$ Ross. ${ }^{1}$ University of Birmingham, Birmingham, UK; ${ }^{2}$ University Hospitals Birmingham, Birmingham, UK

\subsection{6/sextrans-2021-sti.302}

Background The UK National Chlamydia Screening Programme uses an opportunistic approach. Many programmes use campaigns to raise awareness of chlamydia screening in young people. This review aimed to assess the effectiveness of campaigns on uptake of chlamydia screening in young people. Methods We conducted a mixed-methods systematic review of articles assessing the outcomes of community-based health-promotion campaigns to increase chlamydia screening in young people, their experiences of the campaigns and other facilitators and barriers to the conduct of the campaigns. We searched 4 databases for quantitative and qualitative studies with no language restrictions.

Main Results From screening 10,329 records, 100 full text articles assessed in detail leading to 20 studies included in the review; 14 quantitative, 2 qualitative and 3 mixed-methods. All studies with quantitative outcomes were before-after study designs or interrupted time series. Relative change 